\title{
Current density on cathode surface of MPD thrusters
}

\author{
T.S. Sheshadri
}

Indexing terms: MPD thrusters, Current density, Cathode surface

\begin{abstract}
An MPD thruster formulation involving coupled aerothermodynamic-electromagnetic equations and including viscous effects is developed and solved. The effect of various input parameters on the radial current density distribution at the longitudinal cathode surface is studied. In general, radial current concentrationsare found at the upstream and downstream ends of the cathode. The upstream current concentration is larger in magnitude and is attributed to large values of the Hall parameter. Higher inlet velocities and densities, lower inlet temperatures and propellants of lower molecular weight, all reduce the upstream current concentration. The downstream current concentration is attributed to large values of the inlet velocity.
\end{abstract}

\section{Introduction}

The magnetoplasmadynamic (MPD) thruster is an electric propulsion device with potential for application in space missions requiring high specific impulse. A typical thruster geometry is shown in Fig. 1. Propellant is

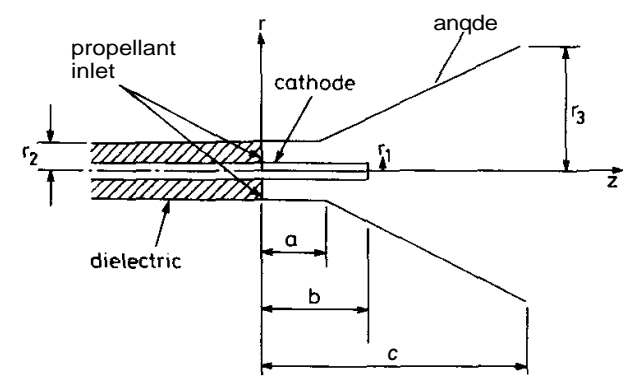

Fig. 1 MPD thruster geometry

injected through inlet slots in the dielectric on the left and is accelerated in the thruster, predominantly by means of the electromagnetic $J \mathbf{x} \boldsymbol{B}$ body force. $B$ is selfinduced by $J$ in the MPD thruster. The study of radial current density distribution on the longitudinal cathode surface is important because a relatively uniform current density distribution suppresses Joule heating loss, improves thrust efficiency and reduces cathode erosion [1]. In previous work, the author has studied the effect of plasma temperature on the current density profile [2], studied the electromagneticforce density distribution [3], and studied the phenomena of onset [4]. In the above References the aerothermodynamic field was assumed known and the electromagnetic equations were solved to obtain results.

In this paper a major generalisation of the above procedure is brought about by simultaneous solution of the coupled aerothermodynamicelectromagneticequations.

\section{Problem geometry. governing equations, solution procedure}

The problem geometry is illustrated in Fig. 1. The details of the electromagnetic equations used and associated boundary conditions are given in Reference 2 . The aerothermodynamic equations considered are the axial momentum equation, the radial momentum equation, the continuity equation and the state equation.

Axial momentum equation

$$
\begin{aligned}
\rho V_{Z} \frac{a V_{\Sigma}}{\partial Z}= & -\frac{\partial P}{\partial Z}+J_{r} B_{0} \\
& +\mu\left[\frac{4}{3} \frac{\partial^{2} V_{Z}}{\partial Z^{2}}+\frac{\partial^{2} V_{Z}}{\partial r^{2}}+\frac{1}{r} \frac{\partial V_{Z}}{\partial r}\right]
\end{aligned}
$$

subject to:

$$
\begin{aligned}
& V_{Z}=\boldsymbol{O} \text { on solid surfaces } \\
& V_{Z}=\text { specified inlet value }\left(V_{\text {in }}\right) \\
& \quad \text { at } Z=0, r_{1}<r<r_{2} \\
& \frac{\partial V_{Z}}{\overline{a Z}}=0 \text { at } Z=C, 0 \leqslant r<r_{3}
\end{aligned}
$$

where $p=$ plasma density, $V_{Z}=$ plasma axial velocity, $\mathrm{Z}=$ axial co-ordinate, $\mathrm{P}=$ pressure, $\boldsymbol{J}_{\boldsymbol{r}}=$ radial current density, $\boldsymbol{B}_{\boldsymbol{\theta}}=$ azimuthal magnetic induction field, $\mu=$ plasma viscosity,$r=$ radial co-ordinate.

The first condition follows from the inclusion of viscosity in eqn 1 . The inlet velocity $V_{i n}$ will have a profile between $r_{1}$ and $r_{2}$ but here it is assumed constant. The last condition is based on the assumption that propellant acceleration is very small at the exit. The developed solution software, however, permits arbitrary profiles of $\boldsymbol{V}_{\text {in }}$ and the replacement of the third condition by any other more realistic assumption.

\section{Radial momentum equation}

$$
\begin{aligned}
\rho V_{Z} \frac{\partial V_{r}}{\partial Z}= & -\frac{\partial P}{\partial r}-J_{Z} B_{\theta}+\frac{\mu}{3} \frac{\partial^{2} V_{Z}}{\partial Z \partial r} \\
& +\mu\left[\frac{4}{3} \frac{\partial^{2} V_{r}}{\partial r^{2}}+\frac{4}{3} \frac{1}{r} \frac{\partial V_{r}}{\partial r}+\frac{\partial^{2} V_{r}}{\partial Z^{2}}-\frac{4}{3} \frac{V_{r}}{r^{2}}\right]
\end{aligned}
$$


subject to

$$
\begin{aligned}
& \boldsymbol{V}_{\boldsymbol{r}}=0 \text { on solid surfaces } \\
& V_{r}=0 \text { at } \mathcal{Z}=0, r_{1}<r<r_{2} \\
& \frac{\partial V_{r}}{\partial z}-0 \text { at } \boldsymbol{Z}=\boldsymbol{C}, \boldsymbol{O} \leq \mathrm{r}<r_{3}
\end{aligned}
$$

where $\boldsymbol{V}_{\mathrm{r}}=$ plasma radial velocity and $J_{Z}=$ axial current density.

Eqns. 1 and 2 are obtained from eqn. 5-53 of Reference 5 under the assumptions $\boldsymbol{V}_{\theta}=0, \partial / \partial \theta=0, \partial / \partial t=0$,

$V_{r} \ll V_{Z}$ and with electromagnetic body forces included. The azimuthal velocity component is not expected to be present as there are no forces in this direction. The axisymmetric geometry of the problem requires $\partial / \partial \theta=0$ and steady flow implies $\partial / \partial t=0$. The nature of the geometry is such that large values of $V_{r}$ are not likely and the radial electromagnetic force is predominantly balanced by the radial pressure gradient, hence the assumption $V_{r} \ll V_{Z}$

\section{Continuity}

$$
\frac{\partial \rho V_{r}}{\partial r}+\frac{\rho V_{r}}{r}+\frac{\partial \rho V_{Z}}{\partial Z}=0
$$

subject to

$$
\begin{gathered}
\rho=\text { specified inlet value }\left(\rho_{\text {in }}\right) \\
\text { at } Z=0, r_{1}<\mathrm{r}<r_{2} \\
\partial \rho-=0 \text { at } Z=C, 0 \leqslant \mathrm{r}<r_{3} \\
\partial Z=0
\end{gathered}
$$

\section{State}

$$
\boldsymbol{P}=\rho R^{\circ} T / W
$$

where $R^{0}=$ universal gas constant, $T=$ temperature and $W=$ propellant molecular weight.

The solution software requires values of viscosity $\mu$ at each of the grid points. In general, the viscosity is dependent on the species and any two thermodynamic variables, e.g., $\boldsymbol{\mu}=\mu$ (species, temperature, density). The author was not able to obtain the above data at the temperatures and densities under consideration. Furthermore, it was thought unwise to attempt a solution of the most general case. Consequently, viscosity is considered as an independent parameter which is assigned a constant value at all grid points without regard to the temperature, density and species. To this extent the solutions obtained do not depict real gas effects. However, the main effect of viscosity in this problem is to slow down the flow and it can be expected that the results obtained would be qualitatively valid.

There is an important respect in which the present work differs from that of Reference 2 . Reference 2 uses density independent electrical conductivity data. The present work obtains density as part of the solution and there are many grid points where the density is very low. At these points the electrical conductivity cannot be independent of density. Electrical conductivity at these points is scaled down using the relation $\sigma / \sigma_{0}=\rho / \rho_{0}$ where $\sigma_{0}$ and $\rho_{0}$ are the electrical conductivity and density, respectively, corresponding to the point when electrical conductivity first becomes density dependent. This is consistent with the theory in Reference 6 and is important in understanding and interpreting the results.
Eqns. 1-4 of this paper plus the eqns. 1-8 of Reference 2 were solved simultaneously by repeated iteration of all the equation solver subroutines starting with initial approximations for all variables. Since the equations constitute an elliptic system, the method of solution used was virtually identical to that of Reference 7. Details of the grid employed are given in Reference 4. Convergence is dependent on the initial approximations used and often extensive trial and error is needed to obtain solutions. Typically the time taken to solve the system of equations to generate one data point was about two to three hours using the 80386-80387 PC AT. The results are accurate to at least two decimal places and, at most points, up to

\begin{tabular}{|c|c|c|}
\hline Parameter & Typical value & Other values \\
\hline Anode, cathode potentials & $\pm 15 V$ & - \\
\hline $\begin{array}{l}a \\
b\end{array}$ & $\begin{array}{l}1.4 \mathrm{~cm} \\
2.4 \mathrm{~cm}\end{array}$ & $\overline{-}$ \\
\hline$c$ & $\begin{array}{l}6 \mathrm{~cm} \\
0.2 \mathrm{~cm}\end{array}$ & - \\
\hline$r_{2}$ & $\begin{array}{l}0.7 \mathrm{~cm} \\
3 \mathrm{~cm}\end{array}$ & - \\
\hline Species & Argon & Hydrogen \\
\hline $\begin{array}{l}\text { Inlet velocity } V, \\
\text { Inlet density } \theta\end{array}$ & $1000 \mathrm{~cm} / \mathrm{s}$ & $2000 \mathrm{~cm} / \mathrm{s}$ \\
\hline $\begin{array}{l}\text { Plasma temperature } T \\
\text { Plasma viscosity } \mu\end{array}$ & $\begin{array}{l}10000 \mathrm{~K} \\
0.00235 \mathrm{gm} / \mathrm{cm} \mathrm{s}\end{array}$ & $\begin{array}{l}8000 \mathrm{~K} \\
0.005 \mathrm{gm} / \mathrm{cm} \mathrm{s}\end{array}$ \\
\hline
\end{tabular}
three decimal places. This has been checked by imposing different values for the magnitude of the maximum error permissible at each grid point. Input parameters required in the solutions along with typical values and the range of variations are listed in Table 1. In the results described below, all input parameters have typical values unless they are being varied.

Table 1 : Inout Darameter values

\section{Results}

The effect of various parameters on the radial current density distribution at the longitudinal cathode surface is considered below

\subsection{Effects of propellant choice}

The effect of propellant choice on the radial current density distribution at the longitudinal cathode surface is shown in Fig. 2. It is clear from Fig. 2 that the upstream current concentration is caused by large values of the Hall parameter. In the present case, since inlet density, temperature, and degree of ionisation are identical for both the propellants, the Hall parameter becomes proportional to molecular weight. This results in a larger upstream current concentration in the case of argon as compared to hydrogen.

\subsection{Effect of inlet velocity $V_{\text {in }}$}

The effect of inlet velocity on the radial current density distribution at the longitudinal cathode surface is shown in Fig. 3. Increasing inlet velocity reduces the upstream current concentration and also causes a current concentration at the downstream end. Over much of the cathode surface the radial current density magnitudes increase significantly due to larger plasma densities caused by the increased mass flow rates.

\subsection{Effect of inlet density $p$,,}

The effect of inlet density on the radial current density distribution at the longitudinal cathode surface is shown in Fig. 4. Increasing inlet density reduces the upstream current concentration by reducing the Hall parameter. 


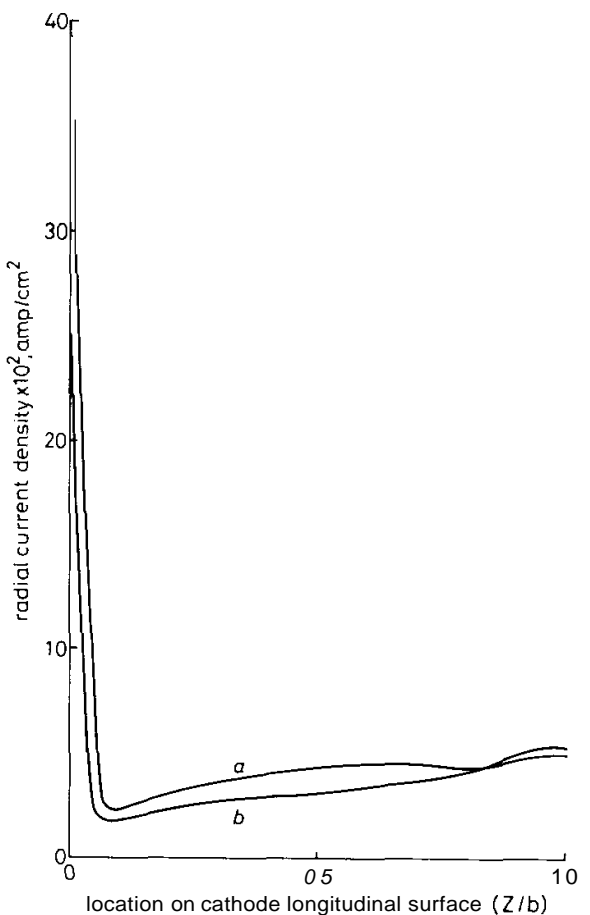

Fig. 2 Effect of propellant choice on radial current density distribution at the cathode longitudinal surface

a Argon $\quad b$ Hydrogen

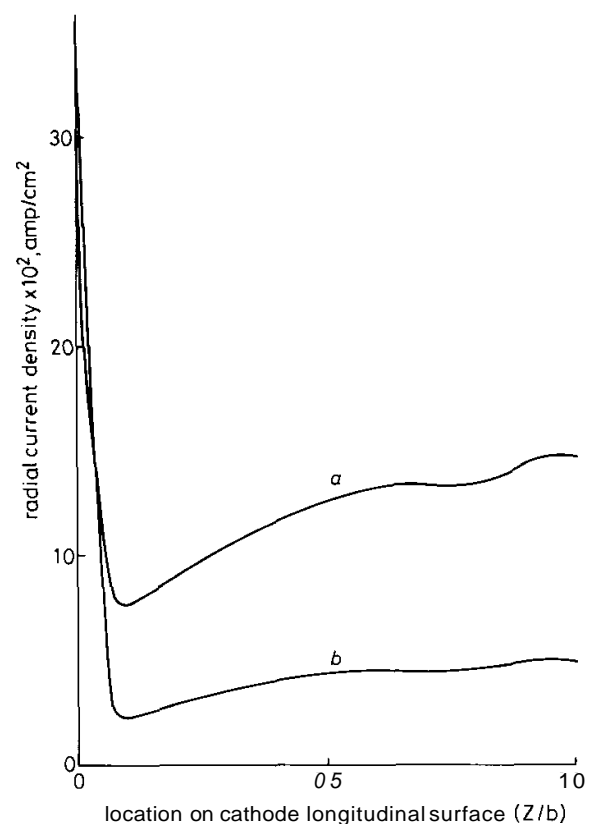

Fig. 3 Effect of inlet velocity on radial current density distribution at the cathode longitudinal surface

a $V_{\text {in }}=2000 \mathrm{~cm} / \mathrm{s}$

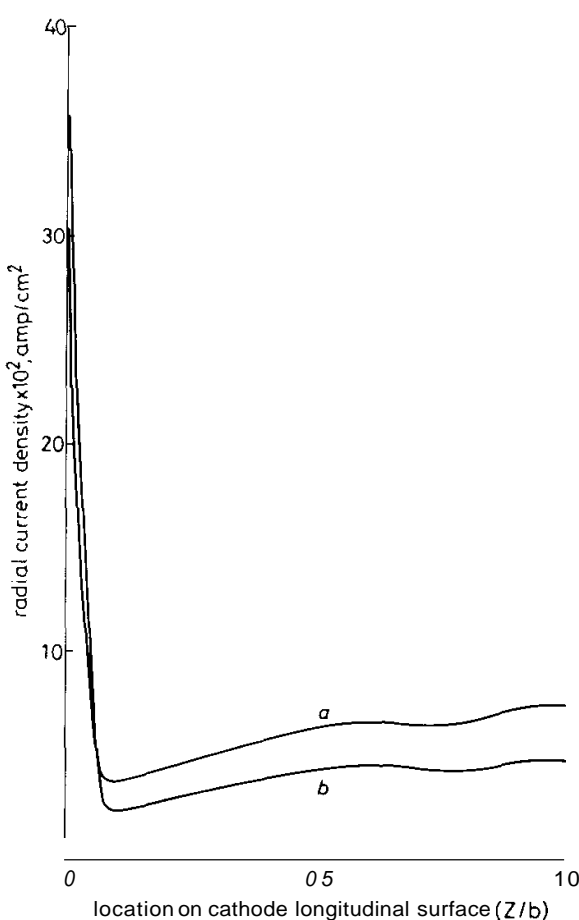

Fig. 4 Effect of inlet density on radial current density distribution at the cathode longitudinal surface

a $\rho_{\mathrm{in}}=\mathrm{I} \times 10^{-5} \mathrm{gm} / \mathrm{cm}^{3}$

b $\rho_{\text {in }}=5 \times 10^{-4} \mathrm{gm} / \mathrm{cm}^{3}$

Radial current density magnitudes increase over the rest of the cathode surface but the effect is not as pronounced as an increase in the inlet velocity.

\subsection{Effect of plasma temperature $T$}

The effect of temperature on the radial current density distribution at the longitudinal cathode surface is shown in Fig. 5. Reduced temperatures lead to a drastic reduction in the upstream current concentration. This is because the Hall parameter is proportional to $T^{\mathbf{1} .5}$. Over the rest of the cathode reduced temperatures lead to a reduction in radial current magnitudes due to lowered electrical conductivities at the lower temperatures.

\subsection{Effect of viscosity}

The effect of viscosity on the radial current density distribution at the longitudinal cathode surface is shown in Fig. 6. Increased viscosities lead to lower plasma velocities and consequently increased plasma densities, resulting in increased current density magnitudes. Increased viscosities lead to an increase in both the upstream and downstream current concentrations.

The above results are important in that they identify the parameters causing the upstream and downstream current density concentrations and also show the effect of the various parameters on the current density over the rest of the cathode longitudinal surface. The results therefore provide qualitative rules which can be used in a practical thruster to reduce upstream and downstream current density concentrations and to uniformly increase current density over the cathode surface so as to bring about better thruster performance. 


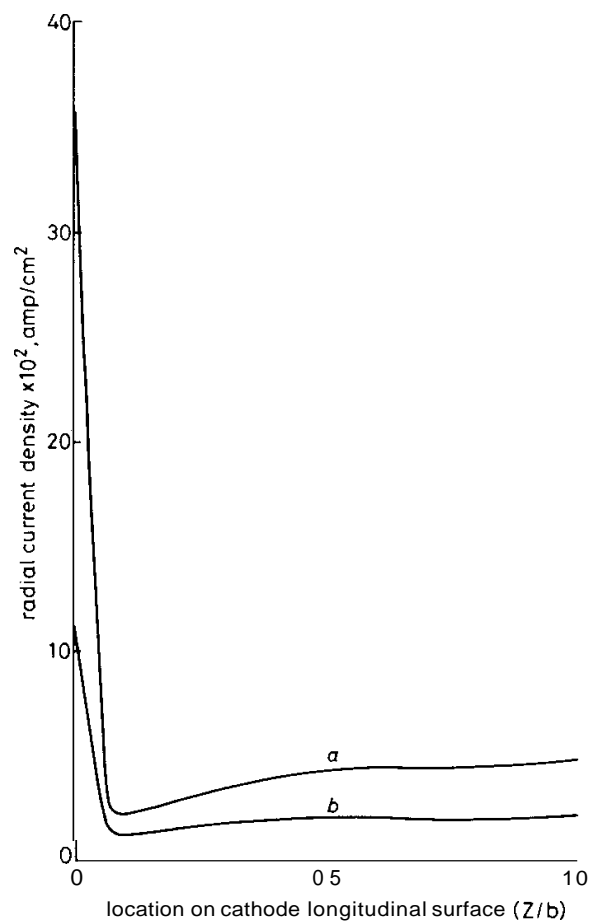

Fig. 5 Effect of temperature on radial cwent density distribution at the cathode longitudinal surface

a Temperature $=\mathbf{1 0 0 0 0 ~ K}$

$b$ Temperature $=8000 \mathrm{~K}$

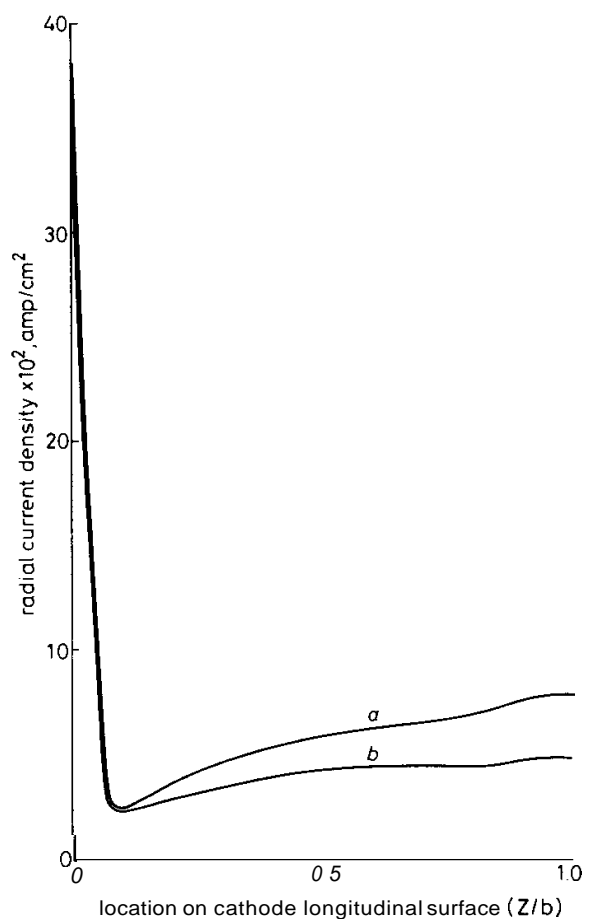

Fig. 6 Effect $f$ viscosity on radial current density distribution at the cathode longitudinal surface

a $\mu=0.5 \times 10^{-2} \mathrm{gm} / \mathrm{cms}$

$b \mu=0.235 \times 10^{-2} \mathrm{gm} / \mathrm{cms}$

\section{$5 \quad$ References}

1 MINAKUCHI, H., and KURIKI, K.: 'Magnetoplasmadynamic analysis of plasma acceleration'. 17th. International Electric Propulsion Conference, Tokyo, 1984, IEPC 84-06

2 SHESHADRI, T.S.: 'Effect of plasma temperature on MPD arcjet cathode surface current density distribution', Jpn. J. Appl. Phys. 2 1990, 29, (8), pp. L1507-L1508

3 SHESHADRI, T.S.: 'Electromagnetic force density distribution in

MPD thrusters', Vacutum, 1991, $\mathbf{2 2}$ (14), pp. 923-925
4 SHESHADRI, T.S.: 'MHD arcjet onset studies', IEE Proc. A, 1991, 138,(5), pp. 286-287

5 YUAN, S.W.: 'Fundamental equations of the flow of viscous compressible fluids in foundations of fluid mechanics' (Prentice-Hall of India, New Delhi, 1988), Chap. 5

6 BROWN, S.C.: 'Elastic collision phenomena', in 'Introduction to electrical discharges in gases' (John Wiley \& Sons, New York, 1966), Chap. 1

7 JACOBS, D.A.H.: The strongly implicit procedure for the numerical solution of parabolic and elliptic partial differential equations' Central Electricity Research Laboratory Note RD/L/N66/72, 1972 